\title{
Tumor oder Entzündung: Differenzierung mittels USPIO-verstärkter Magnetresonanztomografie
}

\section{Cancer and Inflammation: Differentiation by USPIO-enhanced MR Imaging}

Autor

Institut
P. Seyfer

Klinik für Diagnostische und Interventionelle Radiologie, Universitätsklinikum Gießen und Marburg GmbH, Marburg

\author{
Key words \\ - USPIO \\ - contrast media \\ - MRI \\ - cancer \\ - inflammation
}

(c) This Article was first published in: Seyfer P, Pagenstecher A, Mandic R, Klose KJ, Heverhagen JT. Cancer and inflammation: differentiation by USPIO-enhanced MR imaging. J Magn Reson Imaging 2014 Mar; 39 (3): 665 -672; doi: $10.1002 / j m r i .24200$. Epub 2013 May 30. John Wiley \& Sons Inc. The copyright permission was granted by John Wiley \& Sons, Inc. on Sep 15, 2014

\section{Bibliografie}

DOI http://dx.doi.org/

10.1055/s-0034-1385362

Online-Publikation: 2014

Fortschr Röntgenstr 2014; 186:

1140-1148 @ Georg Thieme

Verlag KG Stuttgart · New York . ISSN 1438-9029

\section{Korrespondenzadresse} Dr. med. Perla Seyfer

Klinik für Diagnostische und Interventionelle Radiologie, Universitätsklinikum Gießen und Marburg GmbH Standort Marburg

Baldingerstraße

35043 Marburg

seyfer@med.uni-marburg.de

\section{Zusammenfassung}

$\nabla$

Ziel: Ziel dieser Arbeit war zu überprüfen, ob Ultrasmall Superparamagnetic Iron Oxide (USPIO) die Unterscheidung zwischen entzündlichen und malignen Veränderungen ermöglichen

Material und Methoden: Die Studie wurde von der örtlichen Tierschutzkommission (Regierungspräsidium in Gießen, Deutschland) gemäß dem deutschen Gesetz für Tierschutz bewilligt und nach den Vorschriften der FELASA durchgeführt. Weißen Neuseelandkaninchen wurde zum einen ein Tumor durch subkutane Implantation und zum anderen eine akute Infektion durch ebenfalls subkutane Applikation von Staphylokokkus aureus induziert. Den Tieren wurde dann das USPIO appliziert und die Signaländerungen sowohl in Tumor als auch Entzündzung mittels MRT beobachtet.

Ergebnisse: Auf den T2*-gewichteten USPIOverstärkten MR-Aufnahmen konnte 24 Stunden nach USPIO-Injektion eine Abnahme der Signalintensität in den Entzündungsgebieten festgestellt werden, während sie in den Tumoren konstant blieb. Auch die entzündeten Bereiche des Tumors zeigten ein ähnliches Verhalten wie das der Entzündungen. Sie reicherten eisenhaltige Makrophagen an, und demonstrierten eine Abnahme der Signalintensität mit der Zeit.

Schlussfolgerung: Unsere Ergebnisse zeigen, dass mittels USPIO-verstärkter MRT zwischen benignen entzündlichen und malignen Veränderungen differenziert werden kann.

Kernaussagen:

- $2^{*}$-gewichtete MR-Sequenzen ermöglichen eine genaue Darstellung der Verteilung von Makrophagen in Läsionen nach i.v. - Gabe von USPIO.

- Eine Differenzierung zwischen malignen und benignen Läsionen ist mittels USPIO möglich.

- Ferumoxtran-verstärkte MR-Bildgebung hat einen potenziellen diagnostischen Wert bei der Unterscheidung von Karzinomen und Abszessen.

\section{Abstract \\ $\nabla$}

Purpose: To assess ultrasmall superparamagnetic iron oxide particles (USPIO) -enhanced MR imaging for the differentiation of malignant from benign, inflammatory lesions.

Materials and Methods: In this study, approved by the local animal care committee, VX2 carcinoma and intramuscular abscesses were implanted into the hind thighs of New Zealand White rabbits. MR imaging was performed pre contrast and serially for $24 \mathrm{~h}$ after the injection of USPIO. MR findings were compared with histopathologic results based on Prussian blue stains for the presence of iron.

Results: Twenty-four hours after the Ferumoxtran-injection, no changes were observed in VX2 carcinomas, whereas a mean reduction of the contrast-to-noise ratio (CNR) of approximately $90 \%$ was noticed in abscesses as well as in necrotic tumors. On histopathologic examination, abscess and necrotic parts of the tumor were found to include iron-containing monocytes demonstrating that the reduction in CNR was caused by USPIO-tagged monocytes.

Conclusion: Our results prove the ability of USPIO-enhanced MRI to differentiate benign, inflammatory from malignant lesions.

Key Points:

- The distribution of phagocytic-active macrophages may be detected by $2^{*}$-weighted images after USPIO injection.

- USPIO allow the differentiation between malignant and benign lesions.

- Ferumoxtran-enhanced MRI has a value as a diagnostic tool for differentiating carcinoma from abscesses. 


\section{Einleitung}

\section{$\nabla$}

Die Differenzierung von benignen und malignen Läsionen, v.a. des gastrointestinalen Traktes, ist weiterhin eine unbewältigte Herausforderung für die nicht invasiven diagnostischen Modalitäten. Moderne Schnittbildverfahren können zwar - mehr oder weniger gut - größere Abszesse von malignen Läsionen unterscheiden, sie stoßen allerdings oft an ihre Grenzen, wenn es um die Differenzierung von kleineren Läsionen geht, v. a. im Darm. Beim Vorhandensein größerer entzündlicher Prozesse, beispielsweise der Divertikulitis, kann eine zugrunde liegende maligne Ursache nicht ausgeschlossen werden. Eine Unterscheidung dieser beiden Entitäten ist jedoch notwendig, um eine entsprechende therapeutische Entscheidung treffen zu können, hierbei kann teilweise eine diagnostische Laparotomie erforderlich sein. Ein inflammatorischer Prozess kann konservativ mittels Antibiotika therapiert werden, wohingegen ein Malignom nur durch ein radikaleres Vorgehen behandelt werden kann.

Die Kernspintomografie (MRT) hat durch die kontinuierlichen technischen Weiterentwicklungen und durch die zunehmende klinische Erfahrung der letzten Jahre einen hohen diagnostischen Stellenwert erreicht. Sie ermöglicht eine sehr gute Darstellung von Weichteilgewebe und liefert detailgenaue Bilder von den unterschiedlichen anatomischen Strukturen. Allerdings stößt die MR-Tomografie derzeit bei der Unterscheidung einer Entzündung bzw. eines Abszesses von einem Karzinom an ihre Grenzen. Zur Verbesserung der Läsionsdarstellung und der Gewebstypisierung bzw. zur Erhöhung der Weichteilkontraste und zwecks einer verbesserten Gewebecharakterisierung mittels der MRT ist die Verwendung von verschiedenen Kontrastmitteln und von besonderen Erfassungstechniken notwendig.

Derzeit ermöglicht eine kontrastmittelverstärkte MRT einen exakteren bzw. sensitiveren Nachweis von raumfordernden Prozessen sowie die differenzialdiagnostische Abgrenzung von benignen und malignen fokalen Läsionen. Allerdings ist die Unterscheidung zwischen einer Entzündung und einem malignen Prozess mittels einer kontrastmittelverstärkten MRT nicht immer möglich, da oft beide Läsionen eine verstärkte Vaskularisation nachweisen.

Ultrasmall superparamagnetic iron oxide particles (USPIO) erzeugen in $\mathrm{T}^{*}$-gewichteten Bildern eine Abnahme der Signalintensität [1 - 5]. Deshalb werden sie auch als „negative Kontrastmittel“ bezeichnet. SPIOs werden von den Organen des retikuloendothelialen Systems (RES) und von Makrophagen aufgenommen [6]. Demzufolge kontrastieren sie die Lymphnoten, die normal funktonierende Makrophagen enthalten. Mehrere Studien haben bereits gezeigt, dass die USPIO-verstärkte MRT bei der Detektion von Lymphknotenmetastasen mehrerer Karzinome beispielsweise Brustkrebs, Larynxkarzinom, Prostatakarzinom und Harnblasenkarzinom effizient ist [7-12]. Allerdings haben, nach unserem besten Wissen, noch keine experimentellen Studien, die Fähigkeit von USPIOs bei der Differenzierung zwischen Abszessen von malignen Tumoren untersucht.

Makrophagen spielen eine zentrale Rolle in der Pathogenese von Entzündungen, die durch bakterielle Infektionen verursacht werden. USPIO-Partikel werden durch die Makrophagen aufgenommen. Die nach USPIO-Injektion eisenhaltigen Makrophagen führen zu Signalveränderungen bzw. zu Veränderungen der magnetischen Eigenschaften des Gewebes, die mittels der Kernspintomografie detektiert werden können. Mehrere In-vitro- und In-vivo-Studien in Tieren und Menschen haben bereits gezeigt, dass die Detektion von Makrophagen mittels MRT nach intrave- nöser Injektion von USPIOs eine hohe Aussagekraft hat [5, 6, 11, 13 -20]. Demzufolge ist eine MRT-basierte Visualisierung der Makrophagenaktivität in Raumforderungen für klinische Zwecke wünschenswert. Eine Technik, die in der Lage ist, phagozytierende Makrophagen mittels eisenoxidassoziierter Signaleffekte in vivo zu detektieren, könnte die Unterscheidung zwischen einem malignen Tumor und einer benignen Entzündung ermöglichen.

Diese Studie wurde zur Evaluierung der diagnostischen Aussagekraft der USPIO-gewichtete MR-Bildgebung bei der Differenzierung zwischen Entzündungen und Tumoren, in einem Tiermodel, das beide enthält, durchgeführt. Diese wurde als Proofof-Concept-Studie angelegt.

\section{Material und Methoden \\ $\nabla$}

\section{Implantation von Karzinom und Entzündung}

Die im Folgenden dargestellte Studie wurde von der örtlichen Tierschutzkommission (Regierungspräsidium in Gießen, Deutschland) gemäß dem deutschen Gesetz für Tierschutz bewilligt und nach den Vorschriften der FELASA durchgeführt.

Die Studie wurde an 11 gesunden, ausgewachsenen, männlichen weißen Neuseelandkaninchen mit einem Gewicht von ca. 3 - $5 \mathrm{~kg}$ durchgeführt.

Der VX2-Tumor wurde als Tumormodell ausgesucht, aufgrund seines schnellen Wachstums und seiner einfachen Identifikation mittels MRT. Zunächst wurde zur initialen Tumorimplantation eine tiefgefrorene VX2-Vitalsuspension aus dem Labor der HalsNasen-Ohren-Klinik Marburg, Deutschland benutzt. Durch wiederholte Inokulation, Extraktion und Aufbereitung der VX2Karzinome war es möglich, ausreichende Mengen einer VX2-Vitalsuspension zur späteren Tumorinduktion zu erhalten.

Die Tumorimplantation erfolgte zwei Wochen vor Durchführung der Bildgebung. Jedem Versuchskaninchen wurden zur Inokulation jeweils $0,7 \mathrm{ml}$ einer $10^{7} / \mathrm{ml}$ VX2-Zellsuspension mittels einer 21Gauge-Nadel randomisiert entweder in die rechte oder linke Hinterlaufmuskulatur injiziert. Um einen intramuskulären Abszess in der jeweils kontralateralen Flanke zu erzeugen, wurde ein mit ca. $10^{6}$ Staphylococcus aureus beimpftes Vacuum Assisted ClosureTherapy (VAC)-Schwammstück 7 - 10 Tage nach Tumorinokulation in die dorsale Flankenmuskulatur implantiert. Die Staphylococcus aureus Bakterien zur Abszesserzeugung stammten aus dem Institut für Medizinische Mikrobiologie und Krankenhaushygiene der Philipps Universität in Marburg, Deutschland.

Um den optimalen Zeitpunkt für die Fortsetzung des Versuches festlegen und Tumore von ausreichender Größe für die Untersuchungen auswählen zu können, wurden regelmäßige Tumorgrößenkontrollen mittels klinischer Beobachtung und Palpation durchgeführt. Bei einem nach 7 Tagen ca. $1-2 \mathrm{~cm}$ großen tastbaren Befund wurden die Tiere in die Studie eingeschleust und es wurde ihnen nachfolgend ein Abszess in die gegenseitige Hinterflanke implantiert. Nach Beenden der MRT-Messungen wurden die Kaninchen zur Durchführung des radiologisch-histologischen Vergleiches euthanisiert ( $\triangle$ Abb. 1).

\section{Anästhesie}

Die Magnetresonanztomografie wurde unter Anästhesie durch eine intramuskuläre Mischinjektion aus Ketamin-Hydrochlorid (50 mg/KG; Ketavet ${ }^{\circledR}$, Pfizer, Karlsruhe, Germany), Xylazine (5 mg/ $\mathrm{kg}$; Rompun ${ }^{\circledR} 2 \%$, Bayer, Leverkusen, Germany) und Glycopyrroniumbromid (0.1 mg; Robinul ${ }^{\circledR}$, Riemser, Greifswald, Germany) durchgeführt ( $\bullet$ Abb. 1). 


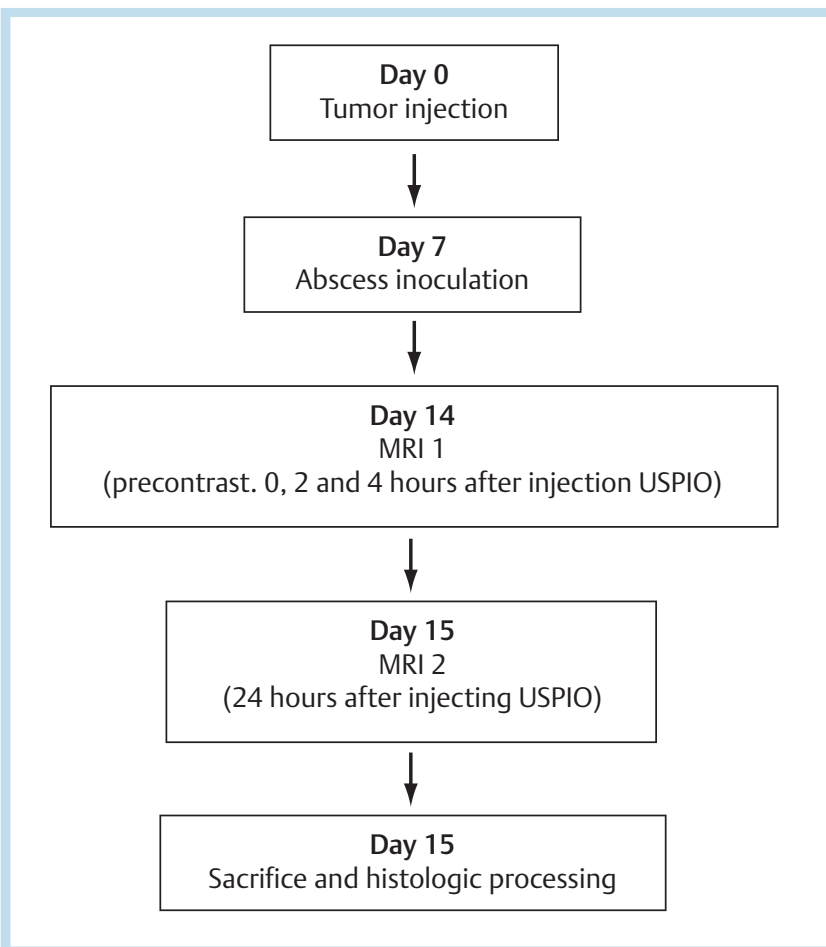

Abb. 1 Zeitlicher Ablauf des Versuches.

\section{MR-Kontrastmittel}

Ferumoxtran-10 ist ein USPIO-Kontrastmittel, das sich zum Zeitpunkt des Studienbeginns in der Phase III der klinischen Erprobung für die Detektion von Lymphknotenmetastasen befand und zum Makrophagenimaging entwickelt wurde [7, 9, 11]. Die USPIO-Partikeln sind mit Dextran beschichtet und haben einen hydrodynamischen Durchmesser von ca. $30 \mathrm{~nm}[14,18]$.

Das USPIO-Kontrastmittel wurde als Bolus direkt nach Durchführung der nativen MR-Sequenzen durch einen intravenösen $\mathrm{Zu}$ gang in die Ohrvene verabreicht.

Eine intravenöse Dosis von $360 \mu \mathrm{mol} \mathrm{Fe} / \mathrm{kg}$ KG wurde jedem Tier, entsprechend der Dosisempfehlung des Herstellers für Kaninchen, verabreicht. Dies entspricht einer Dosis von 0,89 ml Ferumoxtran pro kg KG.

\section{MR-Bildgebung}

Alle tierexperimentellen bildgebenden MR-Untersuchungen wurden bei einer Feldstärke von 1,5 Tesla an einem Magnetom Sonata (Siemens AG, Erlangen) und mit einer zirkulär polarisierten Extremitätenspule (Kniespule) mit $20 \mathrm{~cm}$ innerem Durchmesser durchgeführt.

Die Sequenzparameter für die USPIO-verstärkte MRT beinhalteten eine T1-gewichtete Spinecho (Repetitionszeit (repetition time: TR): $525 \mathrm{msec}$, Echozeit (time to echo: TE): $15 \mathrm{msec}$, Flipwinkel (Flip angles: $\mathrm{FA}$ ): $90^{\circ}$, Schichtdicke (section thickness: $\mathrm{SL}$ ): $2 \mathrm{~mm}$, Schichtabstand (slice distance: SD): 0,4 mm, Bildausschnitt (Field of View: FOV): $100 \mathrm{~mm}$, Matrix: $256 \times 256$ Pixel), eine T2-gewichtete Spinecho (TR: $3000 \mathrm{msec}$, TE: $85 \mathrm{msec}$, FA: $180^{\circ}$, SL: $2 / 2,4 \mathrm{~mm}$, FOV: $100 \mathrm{~mm}$, Matrix: $256 \times 256$ Pixel) und T2*-gewichtete MR Gradientenechosequenzen (TR: $500 \mathrm{msec}, \mathrm{TE}: 20 \mathrm{msec}, \mathrm{FA}: 1^{\circ}$, SL: 2/2,4 mm, FOV: $100 \mathrm{~mm}$, Matrix: $256 \times 256$ Pixel)

Es wurden axiale Schnitte über beide Hinterläufe akquiriert.

Am Tag 1, erfolgte die native MR-Bildgebung. Die erste Post-KMAufnahme erfolgte unmittelbar nach Ferumoxtran-Gabe. Die
USPIO-gewichteten Aufnahmen erfolgten zum Zeitpunkt 0, 2, 4 und 24 Stunden nach Injektion des Kontrastmittels. Die T1, T2 und $\mathrm{T}^{*}$-gewichteten Sequenzen wurden in identischen räumlichen Ausrichtungen durchgeführt.

Die erste Kernspintomografie erfolgte nativ. Die folgenden Untersuchungen waren USPIO-verstärkt. Die Aufnahme der Post-KMBilder für die Untersuchungsstudie (T1, T2 und T2*) begann mit der Verabreichung des Kontrastmittels. Insgesamt wurden bei jedem Tier jeweils Aufnahmen zum Zeitpunkt der Kontrastmittelapplikation sowie 2, 4 und 24 Stunden nach Injektion des Kontrastmittels in T1, T2 und T2*-Sequenzen durchgeführt.

Zwischen den Untersuchungen wurden die Tiere aus dem Magneten entfernt und zum Ausruhen in einzelne Liegekartons platziert. Die Anästhesie wurde nicht beibehalten. Die Tiere wurden allerdings kontinuierlich überwacht.

\section{Datenanalyse}

Sowohl die histologische Untersuchung als auch die radiologische Auswertung erfolgten verblindet. Die Untersucher wussten nicht aus welchen Kaninchen das Präparat stammte bzw. ob es sich um den Abszess oder das VX2-Karzinom handelte. Die MRAuswertung eines erfahrenen Radiologen wurde mit der histopathologischen Beurteilung des Präparates durch den Pathologen in Korrelation gebracht.

\section{MRT-Bildgebung}

Die Signalintensitätsveränderungen in allen Sequenzen wurden sowohl in den nativen als auch in den USPIO-gewichteten Aufnahmen durch einen Facharzt für Radiologie mit 12-jähriger MRT-Erfahrung ausgewertet. Alle Bilder wurden digital an einer IMPAX-Workstation (IMPAX, AGFA HealthCare, Mortsel, Belgien) für Bilderarchivierung und Kommunikation befundet.

Zur qualitativen Analyse wurden visuell die T2*-gewichteten nativen mit den kontrastverstärkten MR-Aufnahmen der Abszesse und der VX2-Karzinome verglichen. Für die Durchführung der quantitativen Messungen wurde die Bestimmung der SI mittels definierter "region-of-interest“ (ROI)-Messungen mit einer ROI in dem Zielgewebe (Tumor bzw. Entzündung) sowie in der gesunden Muskulatur durchgeführt. Die Signalintensitäten wurden sowohl in den nativen als auch in den kontrastverstärkten T1-, T2und $\mathrm{T}^{*}$-gewichteten Aufnahmen gemessen. Die Veränderung der Signalintensität nach Kontrastmittelapplikation wurde im Zeitablauf ausgewertet.

Aus den hieraus gewonnen Daten wurden die Signal-zu-RauschVerhältnisse (SNR) und die Kontrast-zu-Rausch-Verhältnisse (CNR) berechnet und als objektivierbares Maß der Bildqualität herangezogen. Die SNRs und die CNRs wurden jeweils für die Abszess- als auch für die VX2-Karzinomgruppen mit den entsprechenden kontrastverstärkten MR-Sequenzen verglichen [19]. SNR (Abszess bzw. Tumor) $=$ SI (Abszess bzw. Tumor)/SD (Muskel). CNR (Abszess bzw. Tumor) $=$ SI ([Abszess bzw. Tumor] SI [Muskel]/SD [Muskel]).

\section{Histopathologische Untersuchung}

Nach dem Ende der Messungen im MRT wurden die Tiere mit einer Überdosis Ketamin-Hydrochlorid euthanisiert. Anschließend wurde das Tumor- bzw. Abszessgewebe entnommen. Die gewonnenen Abszesse und VX2-Karzinome wurden präpariert und in $10 \%$ igem Formaldehyd fixiert. Nach der Fixierung wurden die Präparate in mit den MR-Sequenzen korrelierende 2-3 mm dicke Transversalstücke geschnitten. Die Präparatstücke wurden 

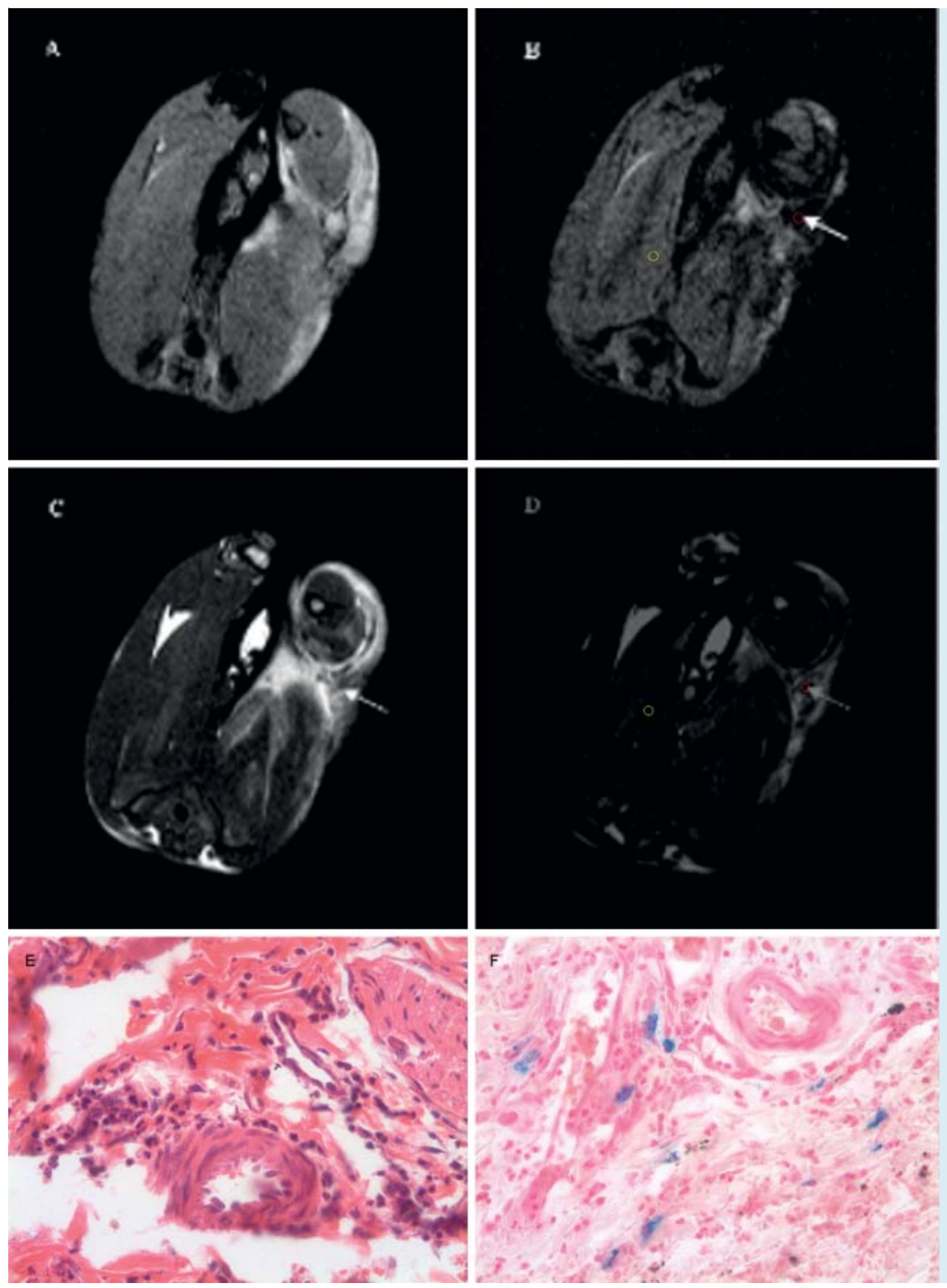

Abb. 2 Abszess in der linken Flanke eines Kaninchens acht Tage nach Inokulation. T2*-gewichtete Aufnahmen vom Abszess nativ (A; TR/TE: 500/20; FA: 15) und 24 Stunden nach USPIO-Injektion (B, C, D). Die T2*-gewichteten Aufnahmen nach USPIO-Gabe B zeigen eine signifikante Abnahme der Signalintensität im Abszess (Pfeil) (rote ROI im Vergleich zur Referenz, die Muskulatur, gelbe ROI). In den T1-gewichteten $\mathbf{C}$ und T2-gewichteten $\mathbf{D}$ Aufnahmen wurde keine signifikante Signalintensitätsveränderung beobachtet. Die histopathologischen Untersuchungen des Abszesses mit Hematoxylin-eosin- $\mathbf{E}$ und Berliner-Blau-Färbung $\mathbf{F}$ zeigen, dass die Hypointensität in den $\mathrm{T} 2{ }^{*}$-gewichteten Aufnahmen durch eine dichte Ansammlung von eisenhaltigen Makrophagen im fibrotischen entzündeten Gewebe verursacht wurde. erneut in $10 \%$ igem Formaldehyd fixiert und später zur weiteren histologischen Aufbereitung in Paraffin eingebettet.

Für die lichtmikroskopischen Untersuchungen der Tumorgewebeproben wurden die Paraffinblöcke mithilfe eines Mikrotoms in $2 \mu \mathrm{m}$ dicke Scheiben geschnitten, auf einen Objektträger aufgetragen und anschließend gefärbt.

Da es sich bei dieser Arbeit um ein eisenhaltiges Kontrastmittel handelt, wurde zur Darstellung der im Gewebe vorhandenen, bzw. aufgenommenen USPIO-Partikeln eine Berliner-Blau-Färbung benutzt. Zur Beurteilung der Tumormorphologie wurde eine Hematoxylin-Eosin-Färbung angewandt.

In der radiologisch-histologischen Korrelationsanalyse wurden die histologischen Präparate mit den USPIO-verstärkten Aufnahmen verglichen. Die mit Berliner-Blau gefärbten Präparate wurden auf das Vorhandensein von Eisenpartikeln in dem Zytosol der Makrophagen untersucht. Der Befund wurde dementsprechend als positiv bzw. negativ ausgewertet.

\section{Statistik}

Die statistische Signifikanz von Unterschieden zwischen den SI, SNR und CNR vor und nach Kontrastmittelinjektion wurde mittels eines Student's-t-test analysiert.

Für alle statistischen Tests galt ein Signifikanzniveau von $\mathrm{p} \leq 0,05$. Die Datenerfassung und statistische Auswertung erfolgte mit SPSS 17.0 (SPSS, Inc. Chicago, IL).

\section{Ergebnisse \\ $\nabla$}

Alle untersuchten Tiere entwickelten einen VX2-Tumor mit einem Durchmesser von mehr als $1 \mathrm{~cm}$ nach einer intramuskulären Inokulation einer VX2-suspension und einen intramuskulären Abszess nach der Implantation eines mit Staphylococcus aureus beimpften VAC-Schwammes. 

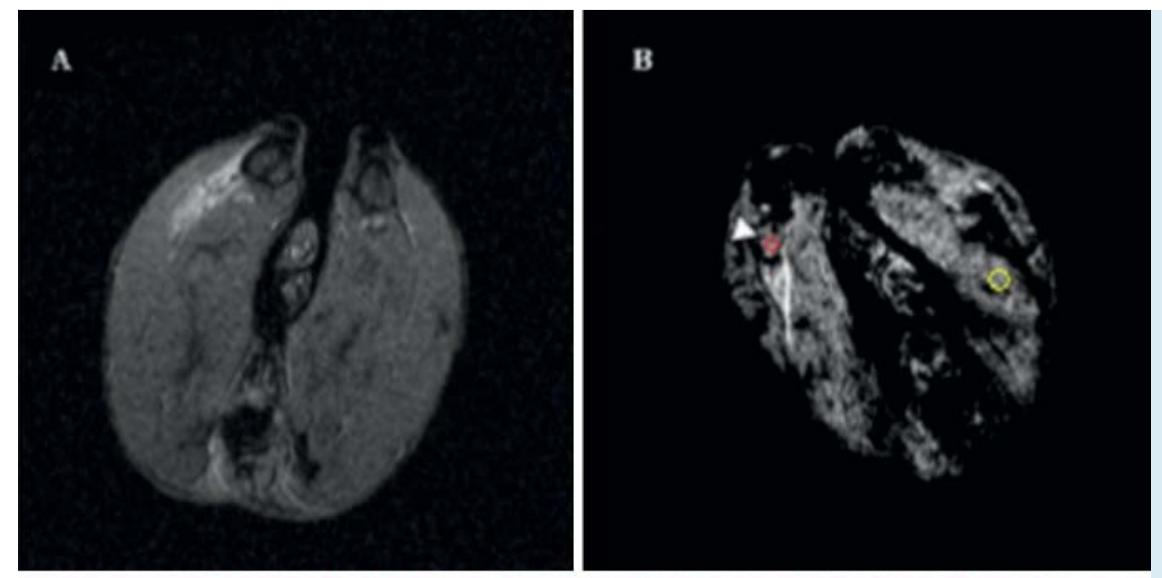

Abb. 3 A-D Vitaler VX2-Tumor in der rechten flanke eines Kaninchens. Transversale T2* -gewichtete Aufnahmen nativ $\mathbf{A}$ und 24 Stunden nach USPIO-Injektion B sowie mit Hematoxylin-eosin C und Berliner-Blau D angefärbten histopathologischen Schnitte aus dem vitalen Tumor. Die nativen $\mathrm{T} 2^{*}$-gewichteten Aufnahmen B zeigen keine signifikante Signalintensitätsveränderung im Tumor (Pfeilspitze) (rote ROI im Vergleich zur Muskulatur, die als Referenz gilt und mit einer gelben ROI markiert ist). Der Tumor bleibt nach KM-Gabe hyperintens. Die mit Hematoxylin-eosin C und mit BerlinerBlau D angefärbten histopathologischen Schnitte zeigen einen stark proliferativen Tumor ohne Eisennachweis.
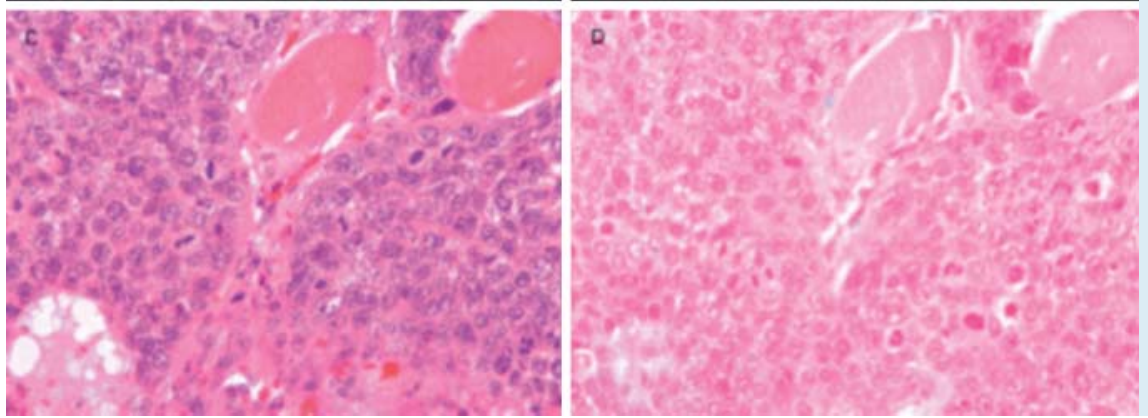

\section{MRT-Auswertung nach Injektion von USPIO} Qualitative Analyse

Keine signifikante Signalintensitätsveränderung wurde in den T1- und T2-gewichteten Aufnahmen beobachtet. Die T2*-gewichtete Aufnahmen zeigten folgendes Signalverhalten:

Die Abszesse waren dargestellt als ovale hyperintense Areale. Eine signifikante Verminderung der SI wurde in den Abszessen unmittelbar nach der Injektion von USPIO festgestellt. Diese nahm 2 und 4 Stunden nach Kontratmittelgabe kontinuierlich ab. Der Abszess stellte sich in allen Tieren nach USPIO-Gabe dunkel dar.

Die SI-Veränderung variierte innerhalb des Karzinoms. Aufgrund des schnellen Tumorwachstums wurden Anteile des Tumors nekrotisch. In den soliden, vitalen Anteilen des Tumors nahm die Signalintensität initial (unmittelbar nach USPIO-Injektion) ab und erholte sich durchgehend nach 2 und 4 Stunden. Diese SI-Veränderung kehrte 24 Stunden nach Kontratmittelgabe vollständig um. In den nekrotischen Tumoranteilen, nahm jedoch die Signalintensität mit der Zeit kontinuierlich ab und erreichte ein Minimun nach 24 Stunden ( $\bullet$ Abb. 2A, B, 3A, B).

\section{Quantitative Messungen der Signalintensität}

Die Signalintensitätsveränderungen in den T1- und T2-gewichteten Aufnahmen waren nicht signifikant ( $\odot$ Tab. 1, 2) (৫ Abb.4). In den T2*-gewichteten Aufnahmen konnten jedoch folgende SIVeränderungen beobachtet werden $(\bullet$ Tab. 3$)$ :

\section{Abszess}

Das Signal-zu-Rausch-Verhältnis (SNR) zeigte in den T2*-gewichteten Aufnahmen eine kontinuierliche und statistisch signifikante $(p<0,05)$ Abnahme in den Abszessen über einen Zeitraum von 24 Stunden nach USPIO-Gabe $(0,94 \pm 0,43$ vs. $17,60 \pm 5,39)$. Ebenso wurde eine signifikante Abnahme der Kontrast-zu-Rausch-Verhältnisse $(\mathrm{CNR})(\mathrm{p}<0,05)$ in den Abszessen in den Aufnahmen nach KM-Gabe (Mittelwert: CNR $-5,74 \pm 1,42$ ) im Vergleich zu den nativen Aufnahmen (Mittelwert: CNR 6,00 $\pm 3,62$ ) beobachtet.
Tab. 1 Die Signalintensität, Kontrast-zu-Rausch-Verhältnisse und Signalzu-Rausch-Verhältnisse von Tumor ( $\mathrm{T}$ ) und Abszess (A) in T1-gewichteten Aufnahmen. ${ }^{1}$

\begin{tabular}{llll|} 
& & nativ & $\begin{array}{l}\text { 24 h nach USPIO- } \\
\text { Gabe }\end{array}$ \\
\hline Signalintensität & T/M & $1,41 \pm 0,9$ & $1,40 \pm 1,20$ \\
\cline { 2 - 4 } & A/M & $1,35 \pm 1,18$ & $1,55 \pm 1,58$ \\
\hline $\begin{array}{l}\text { Kontrast-zu- } \\
\text { Rausch-Verhält- } \\
\text { nisse }\end{array}$ & CNR T & $3,68 \pm 1,52$ & $3,77 \pm 1,43$ \\
\cline { 2 - 4 } $\begin{array}{l}\text { Signal-zu-Rausch- } \\
\text { Verhältnisse }\end{array}$ & SNR T & $12,14 \pm 2,64$ & $5,15 \pm 3,56$ \\
\cline { 2 - 4 } & SNR A & $12,17 \pm 4,25$ & $13,15 \pm 2,98 \pm 3,84$ \\
\hline
\end{tabular}

1 Keine signifikante Veränderung der SI, CNR und SNR vor und 24 Stunden nach USPIO-Gabe $(p>0,05)$.

Tab. 2 Die Signalintensität, Kontrast-zu-Rausch-Verhältnisse und Signalzu-Rausch-Verhältnisse von Tumor ( $\mathrm{T}$ ) und Abszess (A) in T2-gewichteten Aufnahmen. ${ }^{1}$

\begin{tabular}{|lrrr|} 
& & Precontrast & \multicolumn{1}{l}{$\begin{array}{l}\text { Postcontrast } \\
\text { 24 } \mathbf{h} \text { after } \text { in- } \\
\text { jection of USPIO }\end{array}$} \\
\hline Signalintensität & T/M & $5,51 \pm 1,38$ & $4,48 \pm 1,70$ \\
\cline { 2 - 4 } & A/M & $4,22 \pm 0,99$ & $5,17 \pm 1,21$ \\
\hline $\begin{array}{l}\text { Kontrast-zu- } \\
\text { Rausch-Verhältnisse }\end{array}$ & CNR T & $12,17 \pm 1,36$ & $12,70 \pm 2,04$ \\
\hline $\begin{array}{l}\text { Signal-zu-Rausch- } \\
\text { Verhältnisse }\end{array}$ & SNR T & $8,70 \pm 3,51$ & $15,25 \pm 4,67$ \\
\cline { 2 - 4 } & SNR A & $11,40 \pm 3,21$ & $16,35 \pm 1,86$ \\
\hline
\end{tabular}

${ }^{1}$ Keine signifikante Veränderung der SI, CNR und SNR vor und 24 Stunden nach USPIO-Gabe $(p>0,05)$. 

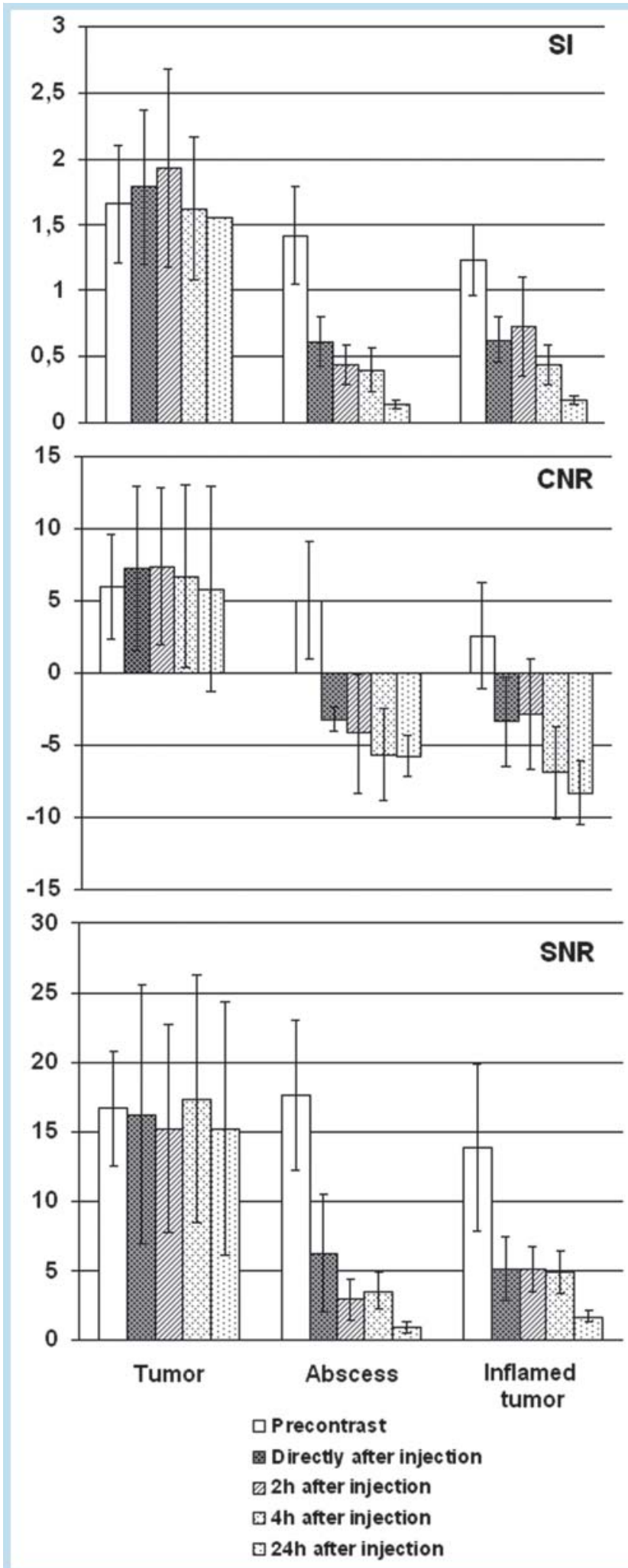

Abb. 4 Signalintensität (SI), Kontrast-zu-Rausch-Verhältnisse (CNR) und Signal-zu-Rausch-Verhältnisse (SNR) im vitalen Tumor, Abszess und in den nekrotischen Tumorarealen 24 Stunden nach USPIO-Injektion (native und Aufnahmen zu den Zeitpunkten 0, 2, 4 und 24 Stunden nach KM -Gabe). Eine signifikante und kontinuierliche Abnahme der SI, CNR und SNR (im Laufe der 24 Stunden nach USPIO-Gabe) wurde in den T2*-gewichteten Aufnahmen von den Abszessen und den nekrotischen Tumoren beobachtet. Die vitalen VX2-Karzinome zeigten keine signifikante Veränderung der Signalintensität nach USPIO-Gabe.
Tab. 3 Vergleich der Signalintensität, Kontrast-zu-Rausch-Verhältnisse und Signal-zu- Rausch-Verhältnisse von vitalem Tumor $(T)$, nekrotischem Tumor (nT) und Abszess (A) in den T2 * -gewichteten MR-Aufnahmen nativ und 24 Stunden nach USPIO-Gabe.

\begin{tabular}{|c|c|c|c|c|}
\hline & & $\begin{array}{l}\text { Precon- } \\
\text { trast }\end{array}$ & $\begin{array}{l}\text { Postcontrast } \\
24 \mathrm{~h} \text { after in- } \\
\text { jection of } \\
\text { USPIO }\end{array}$ & $P$ value \\
\hline \multirow{3}{*}{$\begin{array}{l}\text { Signalin- } \\
\text { tensität }\end{array}$} & $\mathrm{T} / \mathrm{M}$ & $1,66 \pm 0,44$ & $1,55 \pm 0,55$ & $P=0,74$ \\
\hline & $\mathrm{nT} / \mathrm{M}$ & $1,23 \pm 0,27$ & $0,17 \pm 0,01$ & $P<0,05$ \\
\hline & $\mathrm{A} / \mathrm{M}$ & $1,42 \pm 0,37$ & $0,14 \pm 0,03$ & $P<0,05$ \\
\hline \multirow{3}{*}{$\begin{array}{l}\text { Kontrast- } \\
\text { zu-Rausch- } \\
\text { Verhältnisse }\end{array}$} & CNRT & $6,00 \pm 3,62$ & $5,82 \pm 7,09$ & $P=0,96$ \\
\hline & CNR nT & $2,60 \pm 3,63$ & $-8,27 \pm 2,2$ & $P<0,05$ \\
\hline & CNR A & $5,07 \pm 4,06$ & $-5,74 \pm 1,42$ & $P<0,05$ \\
\hline \multirow{3}{*}{$\begin{array}{l}\text { Signal-zu- } \\
\text { Rausch-Ver- } \\
\text { hältnisse }\end{array}$} & SNR T & $16,71 \pm 4,14$ & $15,27 \pm 9,08$ & $P=0,76$ \\
\hline & SNR nT & $13,88 \pm 5,97$ & $1,68 \pm 0,4$ & $P<0,05$ \\
\hline & SNR A & $17,60 \pm 5,39$ & $0,94 \pm 0,43$ & $P<0,05$ \\
\hline
\end{tabular}

Vitaler, solider Tumor

Die 24 Stunden Follow-up-Aufnahmen nach USPIO-Gabe zeigten konstante SNR im Vergleich zu den nativen Untersuchungen in den vitalen Tumoranteilen $(15,27 \pm 9,08$ vs. $16,71 \pm 4,14)$. Die geringen Veränderungen im SNR-Verhältnis waren nicht signifikant $(p=0,76)$. Auch der Mittelwert der CNR-Verhältnisse blieb konstant in den kontrastverstärkten Aufnahmen (Mittelwert: CNR $5,82 \pm 7,09$ ) im Vergleich zu den nativen Aufnahmen (Mittelwert: CNR 6,00 $\pm 3,62$ ). Diese Unterschiede waren demzufolge statistisch nicht signifikant $(p=0,96)$.

\section{Nekrotischer Tumor}

Der Sequenzvergleich der SNR in den entzündeten Tumoranteilen nach der USPIO-Gabe ergab, ähnlich wie bei dem Abszess, eine kontinuierliche und statistisch signifikante $(p<0,05)$ Abnahme der Werte innerhalb der folgenden 24 Stunden $(1,68 \pm 0,4 v$ s. $13,88 \pm 5,97)$. Diese gemessenen Unterschiede waren auch statistisch signifikant $(p<0,05)$. Der CNR-Mittelwert nahm in den nekrotischen Tumorregionenen nach KM-Gabe (Mittelwert: CNR $8,27 \pm 2,20$ ) im Vergleich zu den nativen Aufnahmen (Mittelwert: CNR 2,60 $\pm 3,63$ ) ebenfalls ab. Diese Unterschiede waren ebenfalls statistisch signifikant $(p<0,05)$.

\section{Korrelation zwischen MRT und Histologie}

Die in den hinteren Flanken der Tiere erzeugten Abszesse und Tumore waren in den $\mathrm{T}^{*}$-gewichteten Aufnahmen deutlich von dem normalen Muskelparenchym abgrenzbar.

\section{Abszess}

In den $\mathrm{T}^{*}$-gewichteten kontrastverstärkten Aufnahmen stellte sich der Abszess dunkel dar ( $\boldsymbol{A}$ Abb. 2B). Die korrespondierenden histopathologischen Untersuchungen zeigten, dass die Hypointensität in den MR-Aufnahmen nach Gabe von USPIO durch eine dichte Ansammlung von eisenhaltigen Makrophagen im Gewebe verursacht war. Alle histologisch untersuchten Abszesspräparate wiesen in der Berliner-Blau-Färbung eisenhaltige Makrophagen nach $(\bullet$ Abb.2F).

\section{Vitaler Tumor}

Die kontrastverstärkten Aufnahmen zeigten keine erheblichen Signalveränderungen in den Tumoren im Vergleich zu den Nativaufnahmen. Die Tumore blieben hyperintens in den kontrastverstärk- 

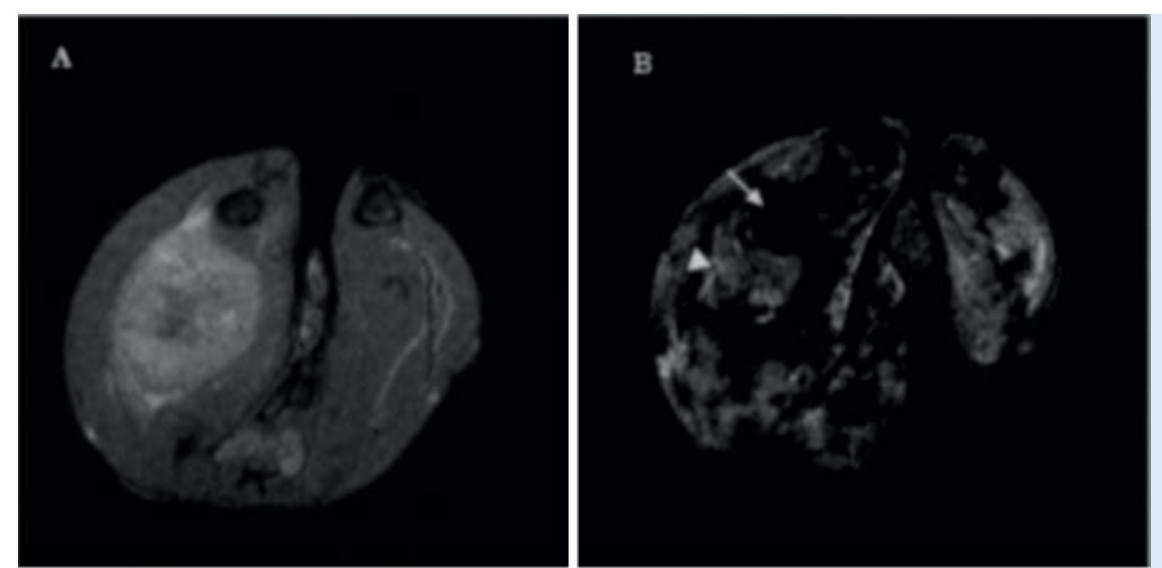

Abb.5 A-D Nekrotischer VX2-Tumor in der rechten Flanke eines Kaninchens. Die transversale T2*gewichtete Aufnahmen (TR/TE: 500/20; FA: 15) von einem VX2 Tumor mit nekrotischen Anteilen (Pfeil) nativ $\mathbf{A}$ und 24 Stunden nach USPIO-Gabe $\mathbf{B}$ zeigen eine signifikante Abnahme der Signalintensität in den nektotischen Anteilen des Tumors (Pfeile) Die vitalen Anteile des Karzinoms weisen keine signifikante Signalintensitätsveränderng (Pfeilspitze) nach. Die Hematoxylin-eosin C und Berliner-BlauFärbungen $\mathbf{D}$ zeigen, in den nekrotischen Anteilen des Tumors, Makrophagen die mit blau gefärbten Eisenoxidpartikeln gefüllt sind. In den vitalen Anteilen des Tumors blieb die Berliner-Blau-Färbung negativ.
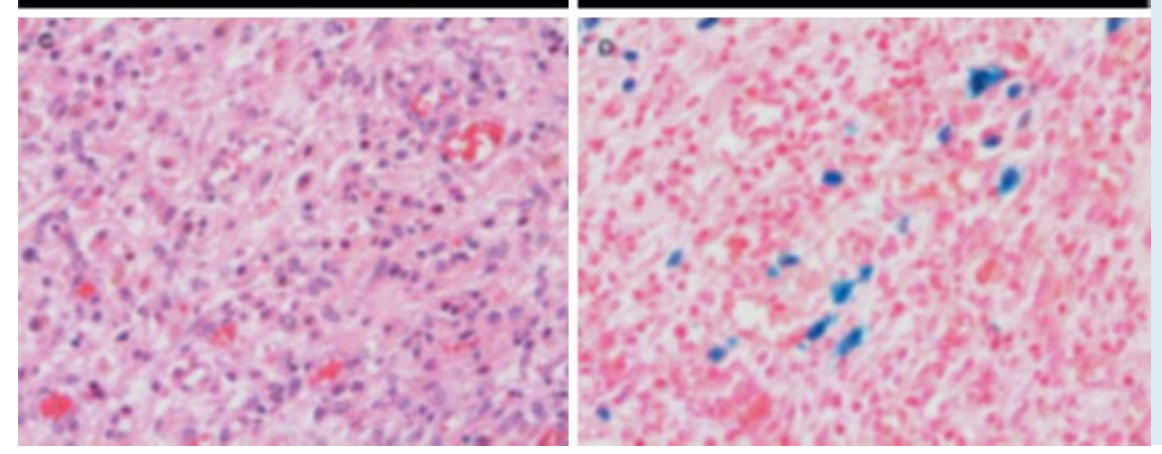

ten T2*-gewichteten Aufnahmen. Die histopathologischen Untersuchungen ergaben keinen Nachweis von eisenhaltigen Makrophagen in den vitalen Tumoren ( $\bullet$ Abb. 3C, D).

\section{Entzündeter Tumor}

Die entzündeten Tumoranteile waren hypointens in den kontrastverstärkten T2*-gewichteten Aufnahmen ( $\bullet$ Abb.5B) und beinhalteten eisenhaltige Makrophagen, die mittels histopathologischen Untersuchungen mit Berliner-Blau-Färbung beobachtet wurden ( $\bullet$ Abb.5D). Demzufolge war die Hypointensität in den entzündeten Tumorbereichen nach KM-Gabe durch eisenhaltige Makrophagen verursacht.

Die histopathologische Untersuchung zeigte, dass die Hypointensität in den Karzinomen nach KM-Gabe mit den nekrotischen Tumorarealen korrelierte und demzufolge die Differenzierung zwischen den nekrotischen und den vitalen Tumoranteilen ermöglichte.

\section{Diskussion}

Die konventionellen bildgebenden Verfahren stoßen an ihre Grenzen, wenn es um die Differenzierung von Läsionen benigner und maligner Genese geht. Diese diagnostische Einschränkung war der Anlass für die Durchführung von Studien an MR-Kontrastmitteln, um ihre Fähigkeit bei der Differenzierung von Tumoren und Abszessen zu untersuchen.

Unter den eisenoxidhaltigen Kontrastmittel, die von Makrophagen phagozytiert werden, sind USPIO-Partikeln für die in vivo makrophagenspezifische MRT besonders gut geeignet [14, 17, 21 -23]. USPIO-Partikel (durchschnittlicher Durchmesser von 18-30 nm) können durch ihren kleinen Durchmesser durch die engen Poren der Gefäßzellen (Durchmesser von 5-100 nm) extravasieren [5, 13, 21]. Nach intravenöser Administration wird ein Teil der injizierten USPIO von Makrophagen und anderen phagozytierenden Zellen, die sich außerhalb der Organe des retikulohistiozytären Systems (RHS) befinden, die vom Entzündungsprozess angezogen werden, aufgenommen [17]. Basierend auf der Tatsache, dass entzündliche Raumforderungen Makrophagen beherbergen, beruht unsere Studie auf der intravenösen Administration von USPIO mit einer langen intravaskulären Halbwertzeit (Ferumoxtran-10). Hierbei sind SI-Veränderungen 2 und 4 Stunden nach KM-Gabe nicht signifikant. 24 Stunden nach Injektion des Kontrastmittels ist die USPIO-Konzentration in den Makrophagen hoch genug um eine Abnahme der Signalintensität hauptsächlich in den $\mathrm{T} 2{ }^{*}$-gewichteten-Aufnahmen zu erzeugen, die mittels klinischer MRTs detektiert werden kann. Die intrazellulären Eisenpartikel in den Makrophagen wurden durch histologische Untersuchungen mit Berliner-Blau-Färbung bestätigt.

Die in dieser Studie durchgeführten qualitativen und quantitativen Analysen zeigen, dass die USPIO-verstärkte MR-Bildgebung dazu geeignet ist, zwischen Karzinomen und Abszessen signifikante Unterschiede von T2*-Signalintensitätsveränderungen zu erfassen. Die beobachtete Signalabnahme in den Abszessen stimmt mit den Ergebnissen vorangegangener Studien überein, in welchen Eisenpartikel für die Abszesssdiagnostik eingesetzt wurden [24-30].

Außerdem zeigten die Ergebnisse unserer Studie, dass die T2*gewichteten MR-Sequenzen eine genaue Darstellung der Verteilung von Makrophagen in Läsionen ermöglichen. Diese Feststellung wurde durch die Korrelation zwischen der histologisch nachgewiesenen Verteilung von aktiven Makrophagen in Abszessen bzw. entzündeten Tumoren und den entsprechenden Signalveränderungen in den $\mathrm{T} 2{ }^{*}$-gewichteten Bildern nachgewiesen. Auch diese Erkenntnis stimmt mit denen aus zahlreichen früheren Untersuchungen, die an SPIO- bzw. USPIO-verstärkter MR-Bildgebung durchgeführt wurden, überein $[5,6,11,15,16$, 18, 20, 22, 26, 27]. Basierend auf den Ergebnissen dieser Studie, 
ist eine zuverlässige Darstellung der Makrophagenaktivität in Entzündungen des Weichteilgewebes mit einem klinischen MR Tomografen möglich.

Wir haben ein Tiermodell untersucht, das sowohl ein VX2-Tumor als auch eine entzündliche Läsion trägt. Nach unserem besten Wissen stellt unsere Studie den ersten direkten Vergleich von Signalintensitätsverhalten in Abszessen und Karzinomen in einem Tiermodell dar. Das Ziel unserer Studie war die Evaluierung der diagnostischen Aussagekraft von USPIOs in der MRT-Bildgebung bei der Differenzierung zwischen Entzündungen und Tumoren. Dies wurde als Proof-of-concept-Studie designed. Wir haben festgestellt, dass subkutane und intramuskuläre Abszesse und Karzinome gut detektierbar und voneinander differenzierbar sind. Darüber hinaus ist aufgrund ihres unterschiedlichen Verhaltens nach USPIO-Injektion eine Unterscheidung zwischen nekrotischen und vitalen Tumoranteilen möglich. Allerdings sind weitere Studien zur Überprüfung der Übertragbarkeit dieses Konzeptes auf die intraabdominellen Tumoren notwendig.

Obwohl die Ergebnisse dieser Studie sehr vielversprechend sind (verbesserte Darstellung von entzündlichen Raumforderungen nach USPIO-Injektion aufgrund der Akkumulation von Makrophagen), sollten auch ihre Limitationen dargestellt werden.

Erstens gehört das VX2-Karzinom zu den aggressiven Karzinomen. Es wächst schnell und entwickelt dementsprechend nekrotische und entzündliche Bereiche. Als Konsequenz dieser schnellen Karzinomwachstumsrate war die durchschnittliche Ausdehnung der VX2-Karzinome größer als die der Abszesse.

Zweitens ließ die bildgebende Analyse erkennen, dass mehrere Karzinome eine Abnahme der Signalintensität und demzufolge eine Abnahme des Signal-zu-Rausch-Verhältnisses (SNR) und des Kontrast-zu-Rausch-Verhältnisses in Teilen der Tumore darstellten, ähnlich wie die Abszesse. Die histopathologischen Untersuchungen zeigten, dass diese Bereiche eisenpositive Makrophagen beinhalteten und dass sie mit den nekrotischen Bereichen der Karzinome korrelierten. Folglich wurden diese nekrotischen Tumorbereiche gesondert analysiert und histopathologisch untersucht bzw. ausgewertet.

Die Identifikation von nekrotischen Tumoranteilen ist von besonderer Bedeutung in Hinblick auf das Monitoring des Tumoransprechens auf eine Therapie. Eine initiale posttherapeutische Größenzunahme des Tumors könnte sowohl durch einen Progress der Grunderkrankung als auch durch die Entwicklung einer Entzündung bzw. einer Nekrose als Folge eines Therapieansprechens entstehen. Lebertumore, welche mittels Radioembolisation oder transarterieller Chemoembolisation behandelt werden, sprechen zuerst mit einer Entzündung (und einer Größenzunahme) an und werden später nekrotisch (und nehmen an Größe ab). Unsere Studie rechtfertigt weitere Untersuchungen zur Evaluierung der diagnostischen Aussagekraft von USPIO-verstärkter MRT bei der Beurteilung des Tumoransprechens auf Therapie. Zusammenfassend hat unsere Studie gezeigt, dass die Verteilung von phagozytoseaktiven Makrophagen in einem experimentell induzierten, bakteriellen Abszessmodel mit einem klinischen MRT nach USPIO-Injektion dargestellt werden kann. Eine Signalintensitätsabnahme in den $2_{2}^{*}$-gewichteten Bildern, wie sie in den Bildern der entsprechenden experimentell induzierten Abszessen nach USPIO-Injektion festzustellen war, ermöglicht eine Differenzierung von vitalen Tumoren und Abszessen. Demzufolge bestätigen die Ergebnisse unserer Studie den Wert der USPIOverstärkten MR-Bildgebung als potenzielles diagnostisches Mittel bei der Unterscheidung von vitalen Karzinomen und Abszessen.

\section{Literatur}

1 Anzai Y, Prince MR. Iron oxide-enhanced MR lymphography: the evaluation of cervical lymph node metastases in head and neck cancer. J Magn Reson Imaging 1997; 7: 75-81

2 Bellin MF, Roy C, Kinkel K et al. Lymph node metastases: safety and effectiveness of MR imaging with ultrasmall superparamagnetic iron oxide particles - initial clinical experience. Radiology 1998; 207: $799-808$

3 Koh DM, Brown G, Temple L et al. Rectal cancer: mesorectallymph nodes at MR imaging with USPIO versus histopathologic finding-initial observations. Radiology 2004; 231: 91 -99

4 Mack MG, Balzer JO, Straub R et al. Superparamagnetic iron oxide-enhanced MR imaging of head and neck lymph nodes. Radiology 2002; 222: $239-244$

5 Weissleder R, Elizondo G, Wittenberg J et al. Ultrasmall superparamagnetic iron oxide: an intravenous contrast agent for assessing lymph nodes with MR imaging. Radiology 1990; 175: 494-498

6 Hauger O, Delalande C, Deminiere C et al. Nephrotoxic nephritis and obstructive nephropathy: evaluation with MR imaging enhanced with ultrasmall superparamagnetic iron oxide-preliminary findings in a rat model. Radiology 2000; 217: 819-826

7 Deserno WM, Harisinghani MG, Taupitz M et al. Urinary bladder cancer: preoperative nodal staging with ferumoxtran-10-enhanced MR imaging. Radiology 2004; 233: 449-456

8 Memarsadeghi M, Riedl CC, Kaneider A et al. Axillary lymph node metastases in patients with breast carcinomas: assessment with nonenhanced versus USPIO-enhanced MR imaging. Radiology 2006; 241: $367-377$

9 Harisinghani MG, Saksena MA, Hahn PF et al. Ferumoxtran-10-enhanced MR lymphangiography: does contrast-enhanced imaging alone suffice for accurate lymph node characterization? Am J Roentgenol Am J Roentgenol 2006; 186: 144-148

10 Nishimura H, Tanigawa N, Hiramatsu M et al. Preoperative esophageal cancer staging: magnetic resonance imaging of lymph node with ferumoxtran-10, an ultrasmall superparamagnetic iron oxide. J Am Coll Surg 2006; 202: 604-611

11 Stets C, Brandt $S$, Wallis $F$ et al. Axillary lymph node metastases: a statistical analysis of various parameters in MRI with USPIO. J Magn Reson Imaging 2002; 16: 60-68

12 Taupitz M, Wagner S, Hamm B et al. Interstitial MR lymphography with iron oxide particles: results in tumor-free and VX2 tumor-bearing rabbits. Am J Roentgenol Am J Roentgenol 1993; 161: 193-200

13 Lutz AM, Seemayer C, Corot C et al. Detection of synovial macrophages in an experimental rabbit model of antigen-induced arthritis: ultrasmall superparamagnetic iron oxide-enhanced MR imaging. Radiology 2004; 233: 149-157

14 Arbab AS, Ichikawa T, Sou H et al. Ferumoxides-enhanced double-echo T2-weighted MR imaging in differentiating metastases from nonsolid benign lesions of the liver. Radiology 2002; 225: 151-158

15 Daldrup-Link HE, Rummeny EJ, Ihssen B et al. Iron-oxide enhanced MR imaging of bone marrow in patients with non-Hodgkin's lymphoma: differentiation between tumor infiltration and hypercellular bone marrow. Eur Radiol 2002; 12: 1557-1566

16 Kanno $S, W u$ YJ, Lee PC. Macrophage accumulation associated with rat cardiac allograft rejection detected by magnetic resonance imaging with ultrasmall superparamagnetic iron oxide particles. Circulation 2001; 104: 934-938

17 Lutz AM, Gopfert K, Jochum W et al. USPIO-enhanced MR imaging for visualization of synovial hyperperfusion and detection of synovial macrophages: preliminary results in an experimental model of antigen induced arthritis. J Magn Reson Imaging 2006; 24: 657-666

18 Metz S, Bonaterra G, Rudelius $M$ et al. Capacity of human monocytes to phagocytose approved iron oxide MR contrast agents in vitro. Eur Radiol 2004; 14: 1851 - 1858

19 Heverhagen JT. Noise measurement and estimation in MR imaging experiments. Radiology 2007; 245: 638-639

20 Rausch M, Baumann D, Neubacher $U$ et al. In-vivo visualization of phagocytotic cells in rat brains after transient ischemia by USPIO. NMR Biomed 2002; 15: 278-283

21 Weissleder R, Cheng HC, Bogdanova A et al. Magnetically labeled cells can be detected by MR imaging. J Magn Reson Imaging 1997; 7 : $258-263$

22 Dousset V, Ballarino L, Delalande C et al. Comparison of ultrasmall particles of iron oxide (USPIO)-enhanced T2-weighted, conventional T2weighted, and gadolinium-enhanced T1-weighted MR images in rats 
with experimental autoimmune encephalomyelitis. AJNR Am J Neuroradiol 1999; 20: 223-227

23 Tatsumi Y, Tanigawa $\mathrm{N}$, Nishimura $\mathrm{H}$ et al. Preoperative diagnosis of lymph node metastases in gastric cancer by magnetic resonance imaging with ferumoxtran-10. Gastric Cancer 2006; 9: 120-128

24 Chan TW, Eley C, Liberti P et al. Magnetic resonance imaging of abscesses using lipid-coated iron oxide particles. Invest Radiol 1992; 27: 443-449

25 Gellissen J, Axmann C, Prescher A et al. Extra- and intracellular accumulation of ultrasmall superparamagnetic iron oxides (USPIO) in experimentally induced abscesses of the peripheral soft tissues and their effects on magnetic resonance imaging. Magn Reson Imaging 1999; 17: $557-567$

26 Kaim AH, Wischer T, O'Reilly T et al. MR imaging with ultrasmall superparamagnetic iron oxide particles in experimental soft-tissue infections in rats. Radiology 2002; 225: 808-814
27 Thorek DL, Weisshaar CL, Czupryna JC et al. Superparamagnetic iron oxide-enhanced magnetic resonance imaging of neuroinflammation in a rat model of radicular pain. Mol Imaging 2011; 10: $206-214$

28 Lutz AM, Weishaupt D, Persohn E et al. Imaging of macrophages in softtissue infection in rats: relationship between ultrasmall superparamagnetic iron oxide dose and MR signal characteristics. Radiology 2005; 234: 765 - 775

29 Triantafyllou M, Studer UE, Birkhauser FD et al. Ultrasmall superparamagnetic particles of iron oxide allow for the detection of metastases in normal sized pelvic lymph nodes of patients with bladder and/or prostate cancer. Eur J Cancer 2013; 49: 616-624

30 Kinner S, Maderwald S, Albert J et al. Comparison of two different iron oxide-based contrast agents for discrimination of benign and malignant lymph nodes. Invest Radiol 2012; 47: 511-515 